\title{
Retraction
}

\section{Retracted: College Physical Education Flipped Classroom Teaching Based on Multimedia Network Data Fusion}

\author{
Wireless Communications and Mobile Computing \\ Received 28 January 2022; Accepted 28 January 2022; Published 6 February 2023 \\ Copyright (c) 2023 Wireless Communications and Mobile Computing. This is an open access article distributed under the Creative \\ Commons Attribution License, which permits unrestricted use, distribution, and reproduction in any medium, provided the \\ original work is properly cited.
}

Wireless Communications and Mobile Computing has retracted the article titled "College Physical Education Flipped Classroom Teaching Based on Multimedia Network Data Fusion" [1] due to concerns that the peer review process has been compromised.

Following an investigation conducted by the Hindawi Research Integrity team [2], significant concerns were identified with the peer reviewers assigned to this article; the investigation has concluded that the peer review process was compromised. We therefore can no longer trust the peer review process and the article is being retracted with the agreement of the Chief Editor.

The author does not agree to the retraction.

\section{References}

[1] M. Zhao, "College Physical Education Flipped Classroom Teaching Based on Multimedia Network Data Fusion," Wireless Communications and Mobile Computing, vol. 2022, Article ID 6533243, 12 pages, 2022.

[2] L. Ferguson, "Advancing Research Integrity Collaboratively and with Vigour," 2022, https://www.hindawi.com/post/advancingresearch-integrity-collaboratively-and-vigour/. 


\title{
College Physical Education Flipped Classroom Teaching Based on Multimedia Network Data Fusion
}

\author{
Meng Zhao \\ College of Physical Education, Yan Shan University, Qinhuangdao, 066004 Hebei, China \\ Correspondence should be addressed to Meng Zhao; zhaomeng1978@ysu.edu.cn
}

Received 24 December 2021; Revised 12 February 2022; Accepted 19 February 2022; Published 7 March 2022

Academic Editor: Rashid A Saeed

Copyright (C) 2022 Meng Zhao. This is an open access article distributed under the Creative Commons Attribution License, which permits unrestricted use, distribution, and reproduction in any medium, provided the original work is properly cited.

\begin{abstract}
In the Internet age, the challenge to traditional physical education classroom teaching has also reached its peak. Traditional physical education teaching has little effect on students. The proposal of flipped classroom can be said to greatly improve the dilemma of traditional physical education classroom teaching. This article is aimed at studying the exploration of flipped classroom teaching in college physical education. For this reason, this paper proposes a data fusion method based on multimedia network to improve the teaching mode and optimizes the algorithm of similar node clustering based on $K$-means, to make this algorithm more suitable for the teaching monitoring of flipped classrooms in college physical education. At the same time, experiments and analysis are designed to explore the effects of flipped teaching mode. The experimental results of this paper show that under the new teaching mode, the initiative of college students in physical education classes has increased by $31 \%$, and their interest in learning in physical education classes has increased by $28 \%$. And after applying the new teaching model, the physical fitness of the students has also improved by $14 \%$, and the mastery of physical skills has become more proficient.
\end{abstract}

\section{Introduction}

With the progress of the technological age, technologies such as the Internet, cloud computing, and resource construction are also undergoing continuous development, and more and more students are more willing to accept learning through the Internet. Let students maintain an active and efficient learning state throughout the entire process of learning a certain sports technical essentials, so as to try to change the current teaching problems of college physical education courses.

Flipped classroom is regarded as a successful paradigm of the organic combination of information technology and education and teaching, and the educational and teaching concepts it contains have also been widely recognized by the education circle. The implementation of flipped classrooms in public physical education in ordinary in China is highly catering to the lifestyle and daily habits of college stu- dents. Students' physical education courses are more natural, interesting, and personalized.

Although the field of physical education in domestic has undergone many reforms, the instillation teaching based on the explanation and demonstration of physical education teachers has not changed substantially. With the introduction of flipped classrooms based on multimedia networks, people began to pay more attention to them. Hasan et al. believe that best-effort data and real-time multimedia application service quality assurance has brought new challenges in the WMSN to give priority to multipath routing protocols [1]. Park et al. studied the mediating role of value in the relationship between the quality of SNS information services and the activities of producers and consumers and discussed the impact of this discovery on future research and practice [2]. Al-Turjman and Radwan believe that a multipath routing protocol with multiple constraints is recommended in WMSN [3]. Lai et al. proposed a cloud multimedia network 
adaptive energy-saving code stream decoding system based on a multicore system [4]. For Jovanović et al., the strategy they learned clusters student files and analyzes them strategically [5]. Baytiyeh believes that student reviews provide evidence for deeper and broader learning perspectives. Research shows that this teaching method can enrich students' learning experience and help them develop the soft skills needed to succeed in any industry [6]. Mcnally et al. believe that although flipped classrooms are popular, their effectiveness in achieving greater participation and learning outcomes currently lacks a lot of empirical evidence [7]. Kostaris et al. believe that the emerging flipped classroom approach has been widely used to strengthen teaching practices in many subject areas and educational levels and reported promising results for improving the learning experience of students [8]. The above-mentioned documents involve related research on multimedia sensor networks and flipped classrooms, and the introduction of related technologies for their respective contents is very well-informed. However, it has not combined the multimedia sensor network with the flipped classroom to conduct a comprehensive research on it.

The innovation of this article lies in the research on the flipped teaching mode of college physical education classrooms by understanding the flipped classroom and using it as the theoretical basis, combined with the technical support of multimedia network data fusion. It also proposes similar node clustering based on $K$-means to measure the data in the movement process and send it to the terminal for statistics, which can well monitor the student's movement status.

\section{Data Fusion Monitoring Method}

2.1. Features of "Flipped Classroom." The wireless-based multimedia network flipped classroom is gradually used frequently due to its convenient characteristics [9-11]. The flipped classroom is translated from "Flipped Classroom" or "Inverted Classroom," which can also be translated as "Inverted Classroom," which refers to readjusting the time inside and outside the classroom, and transferring the decision-making power of learning from teachers to students. The sensor network is shown in Figure 1.

In traditional classroom teaching, teachers have always occupied the main position of education in a dominant form, and students have always been in a passive learning state [12]. In the "flipped classroom" teaching model, the role attribute of classroom teaching is reversed (as shown in Figure 2). Students become the "protagonists" in teaching, and teachers become the "organizers" in teaching [13].

In traditional teaching, when facing fresh knowledge, teachers are more familiar with the content than students, and students learn more passively and have relatively low autonomy. This is what we generally call "cracking duck" teaching. The "flipped classroom" students watch the teaching microvideo before class and to a certain extent have a certain understanding of new knowledge. In this sense, it shows that the degree of mastery of new knowledge by students and teachers is relatively reduced [14]. In the "flipped classroom" teaching, teachers have changed from "leading actors" to "assistant teachers" in the classroom. Students have also changed from passive learning to active learning; not only their thinking is more active, but their roles have also become the "protagonists" of the classroom. Since there is a certain understanding and learning of new knowledge before, it is more integrated in the classroom discussion, and the specific and in-depth questions are asked and tried to solve.

In the traditional teaching classroom, teachers tend to design the curriculum carefully, one by one, and gradually advance the preset mode [15]. Although it can give students the opportunity to express to a certain extent, the final result is still up to the teacher to decide, otherwise the interlocking instructional design cannot be carried out [16]. As a result, teachers' teaching tasks are often unilaterally delivered to students, and students lose more autonomy and thinking learning, and their enthusiasm is greatly reduced. As shown in Figure 3, the classroom mode is flipped from "rigid preset" to "flexible preset" [17].

The essence of "flipped classroom" is that he transformed the rigid form of single-line teaching in the original traditional education model into an educational model with flexible forms. Teachers can carry out the teaching process reasonably according to the situation, which also makes the classroom scene more prominent [18-20].

In the traditional teaching classroom, the teacher presets the teaching content and knowledge points of the whole class when preparing the lesson before class. This is more about the teaching difficulties summarized by the teacher's own teaching experience. The teaching content and methods formulated in this way are the greatest help for students in their studies, and they are more targeted and accurate for students' difficult points in the selfstudy stage [21]. In this way, accurate positioning can effectively help students achieve better learning results (as shown in Figure 4).

In the traditional classroom, the classroom becomes the teacher's "large lecture hall," and the student's task is oneline passive learning. Listening to the lesson-taking notes has become a recurring process, and students easily lose interest in classroom knowledge learning. Internalize in class, and integrate into their own cognitive structure (as shown in Figure 5).

The students in "flipped classroom" use more class time to conduct group discussions and discuss with teachers about their own problems, interests, depth, and value. In this way, the value of classroom teaching can be improved and each student can meet their own needs and increase interest, thereby improving the educational significance of "flipped classroom" in many ways.

2.2. The Concept and Function of Data Fusion. Due to the dense deployment of nodes in wireless multimedia sensor networks, it is very likely that multimedia sensor nodes that are close to each other may collect redundant information [22-24]. If the data collected by each node is completely transmitted to the sink node, it will cause a 


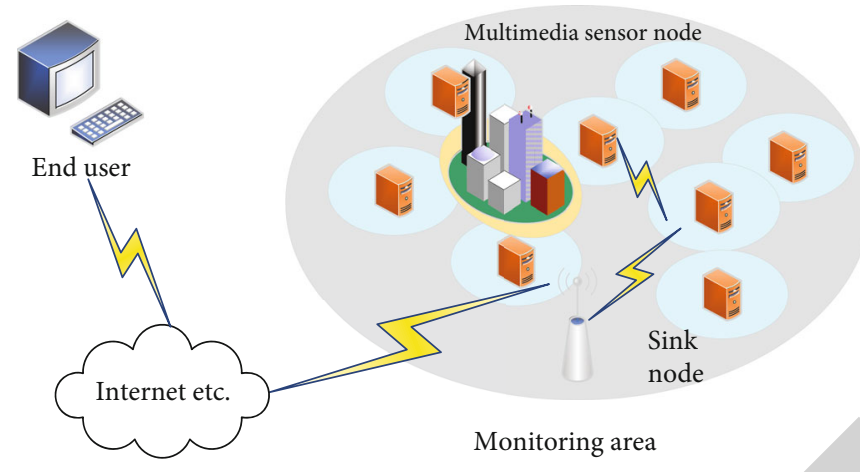

FIgURE 1: Wireless multimedia sensor network.

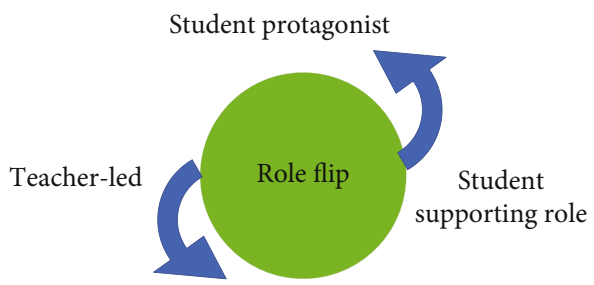

Teacher organization

Figure 2: The role change in the flipped classroom.

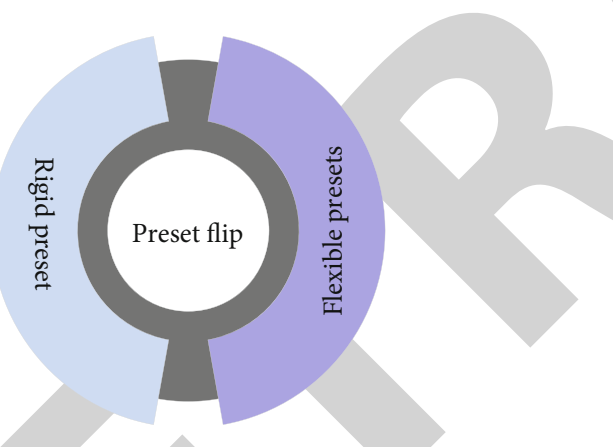

FIGURE 3: The transition from rigid presupposition in traditional teaching to flexible presupposition in flipped classroom.

great waste of communication bandwidth. At the same time, the transmission of a large amount of data will cause the entire network to consume too much energy and shorten the life of the network. The local computing and storage capabilities of multimedia sensor nodes are used to process the collected information, eliminate duplicate and redundant information, reduce unnecessary data transmission, and save the node's transmission energy consumption. In addition, the information collected by different multimedia sensor nodes is compared and analyzed, and the information with large errors collected by individual nodes is excluded, so as to obtain monitoring information with higher reliability.

2.2.1. Saving Network Energy. Due to the dense deployment of nodes in wireless multimedia sensor networks, it is very likely that multimedia sensor nodes that are geographically close to each other may collect redundant data information. If the redundant information is not processed and directly transmitted to the sink node, it will cause a great waste of node energy. Data fusion technology is an effective means to solve this problem, that is, using certain algorithms to eliminate redundant processing of data collected by multimedia sensor nodes, reduce the amount of data that needs to be transmitted, and save the energy consumed by multimedia sensor nodes due to transmission. Then, save the effective energy of the entire network and extend the life cycle of the network.

2.2.2. Enhancing the Accuracy of Data Collection. Since wireless multimedia sensor networks are mostly deployed in unattended environments, they are vulnerable to harsh working environments or the node's own functional components; this causes the node to work abnormally and report incorrect data. Therefore, the data collected by a single multimedia sensor node may have a large error. Data fusion technology can comprehensively analyze the data results collected by multiple multimedia sensor nodes that monitor the same object, eliminate erroneous data, and finally obtain high-precision and high-reliability data results.

2.2.3. Improving the Efficiency of Data Collection. The data collected by geographically close multimedia sensor nodes may have greater redundancy. Transmitting unnecessary data will waste communication bandwidth and consume additional limited energy in the network. Therefore, in the process of wireless multimedia sensor network data collection, the use of data fusion technology can greatly improve some adverse effects.

Generally speaking, the design of application analysis and data fusion is completed according to different levels. Therefore, data fusion can be divided into the following three levels according to the level of fusion, as shown in Figure 6, that is, the fusion types of different levels of original data-level fusion, feature-level fusion, and decisionlevel fusion.

(1) The object of raw data-level fusion processing is the raw data type perceived by multimedia sensor nodes, which belongs to the lowest level of data fusion. In 

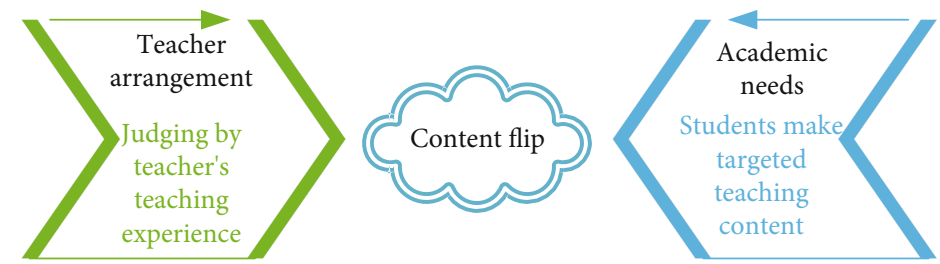

FIGURE 4: The flipping of the teaching content from the rigid preset of traditional teaching to the flexible preset of flipped classroom.

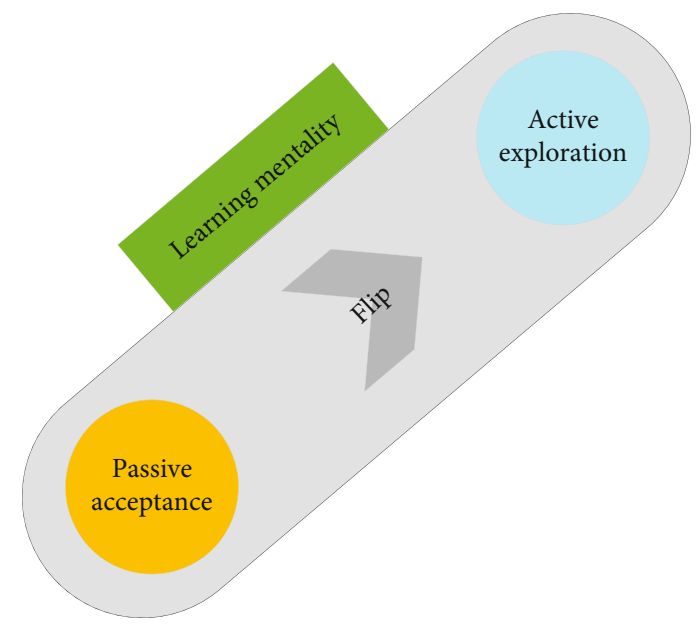

Figure 5: The flip of the student's learning mentality from passive acceptance to active exploration.

most cases, this type of fusion is only related to the type of raw data collected by multimedia sensor nodes and has nothing to do with actual application requirements, so it is a data-oriented fusion. The original data-level fusion is generally applied to data classification and compression

(2) The object of feature-level fusion processing is the characteristics of the data sensed by multimedia sensor nodes, which is the fusion layer between the original data level and the decision-making level. Before performing feature-level fusion processing, the data needs to be expressed as a feature vector that can reflect the attributes of the monitored object, so it is an object-oriented feature data fusion. The key to this level is how to extract stable, consistent, and effective data features. This level of fusion processing will affect the accuracy of the final data collection result due to the loss of a large amount of data

(3) The decision-level fusion processing center classifies and discriminates the features according to the features of the monitored object extracted by the feature-level fusion and finally makes corresponding decisions through certain calculation and processing methods

The data fusion transmission model based on clustering and prediction makes full use of the time and space correlation of sensor data to achieve the purpose of reducing redun- dant data transmission, reducing computing costs, and saving energy. In the data collection and fusion, the terminal and the sensor node should adopt the same data sequence prediction mechanism. The final data node that needs to be transmitted is shown in Figure 7 . The green node whose prediction error is less than the set threshold does not need to send data, and the blue node that fails the prediction can judge whether it needs to send data through the prediction of its connection to the master node. In this way, within the allowable error range, the end user can obtain the data of each sampling point in the sensor node at a lower communication cost.

2.3. Clustering of Similar Nodes Based on K-Means. The purpose of the clustering algorithm is to divide the feature vectors without category marks in the feature space into a certain cluster according to a certain similarity principle in a large-scale data environment, so that the data samples in the same cluster can be distinguished from other clusters. Clustering has strong practicability in emerging fields such as data mining, machine learning, and artificial intelligence. However, the use of LEACH protocol needs to be based on some assumptions, and it is not suitable for large-scale networks and energy imbalanced networks. The $K$-means algorithm has good clustering performance for networks of different sizes. Applying this algorithm to the cluster routing of wireless sensor networks can effectively balance the network load and optimize the overall network performance.

In the sensor network architecture, clustering is one of the effective means to reduce data. The problem with existing node clustering algorithms is that the clustering data is not similar, and the historical data of sensor nodes in a specific period of time can be used as a measure of clustering. Assume that the historical data of each node is

$$
x_{i}=\left[\begin{array}{cccc}
a_{i}(1) & a_{i}(2) & \cdots & a_{i}(t) \\
b_{i}(1) & b_{i}(2) & \cdots & b_{i}(t) \\
\vdots & \vdots & \ddots & \vdots \\
n_{i}(1) & n_{i}(2) & \cdots & n_{i}(t)
\end{array}\right] .
$$

The data matrix can be expressed as a data sequence:

$$
x_{i}=\left[a_{i}(1) \cdots a_{i}(t) b_{i}(1) \cdots b_{i}(t) \cdots n_{i}(1) \cdots n_{i}(t)\right] .
$$

In order to obtain better clustering results, similar data is 


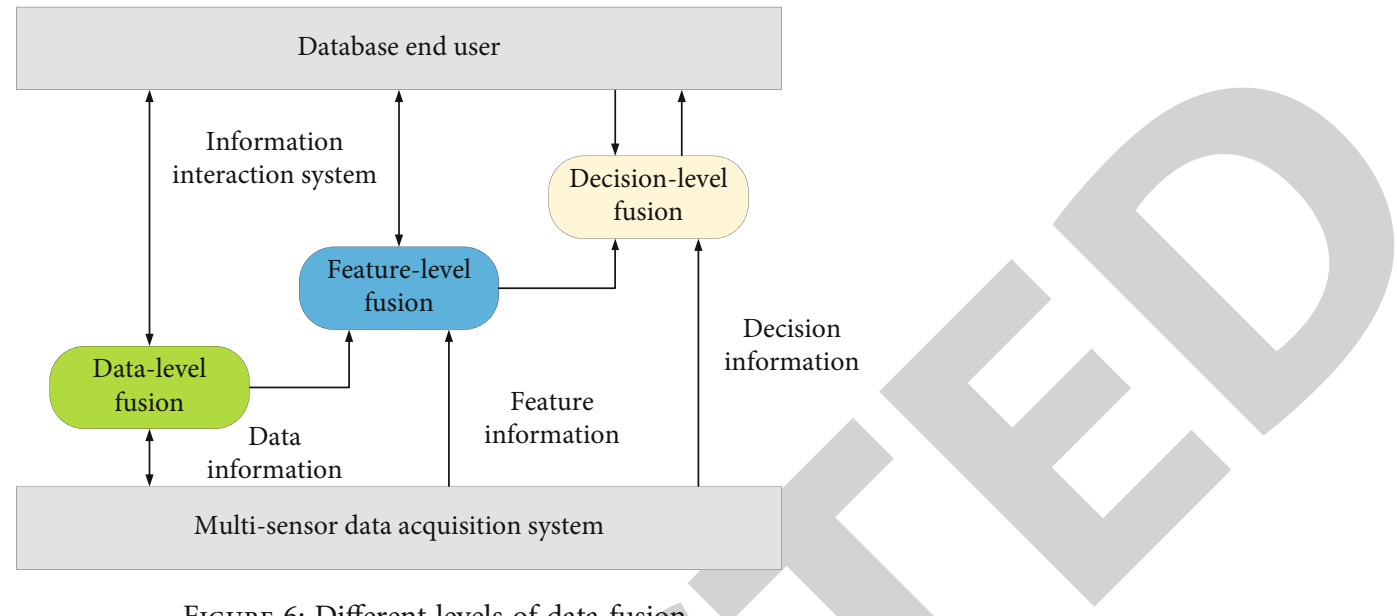

Figure 6: Different levels of data fusion.

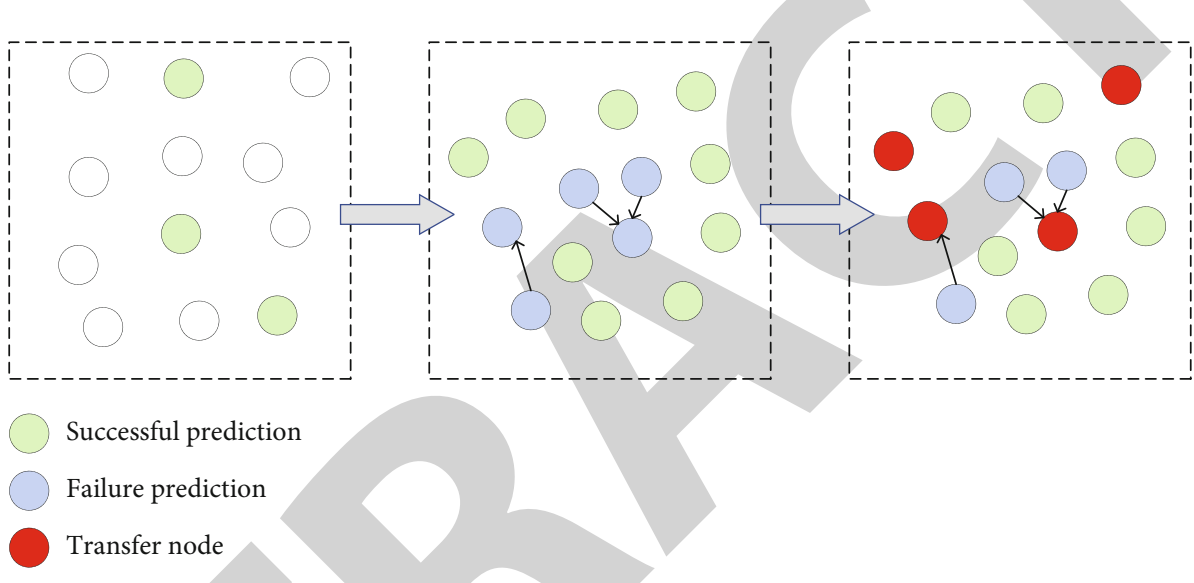

Figure 7: Node transmission diagram.

normalized to the range $[0,1]$ according to the following formula:

$$
s_{t}=\frac{s_{t}-s_{\min }}{s_{\max }-s_{\min }} .
$$

Then, the Euclidean distance between two nodes can be calculated as follows:

$$
\Phi(x, y)=\sqrt{\left(x_{1}-y_{1}\right)^{2}+\left(x_{2}-y_{2}\right)^{2}+\cdots+\left(x_{n}-y_{n}\right)^{2}} .
$$

In the formula, $\Phi_{i j}$ is the similarity between node $i$ and node $j$.

Due to the spatial correlation between the data, some nodes in the area are similar to the data collected by their neighboring nodes. The strong connection also considers the data similarity between nodes and the actual geographic distance, while the weak connection ignores the influence of the actual geographic location on the data. Therefore, strong connections can better eliminate the spatial correlation of data.

After getting the similar connections of the nodes as shown in Figure 8 , the data of the nodes in the strong connection are similar and may even be the same. Select the node closest to the terminal in the strong connection as the master node. When a node needs to transmit data, it only needs to transmit the data to the master node. The master node will calculate the data error of the connected node and determine whether to replace the node data with its own data. The terminal can use the similarity to recover the data of other nodes.

For $N$ training samples $\left(x_{j}, t_{j}\right)$, the output of the extreme learning machine with $L$ hidden layer nodes can be expressed as

$$
\sum_{i=1}^{L} \beta_{i} g\left(\omega_{i} \cdot x_{j}+b_{i}\right)=t_{j}, \quad j=1,2, \cdots, N
$$

In the formula, $\beta_{i}$ is the output weight; $\omega_{i}$ the input weight of the hidden layer node; $b_{i}$ the bias value of hidden layer node; and $g(\cdot)$ the nonlinear activation function.

Therefore, the above formula can be further compactly expressed as a matrix:

$$
H \beta=T \text {. }
$$




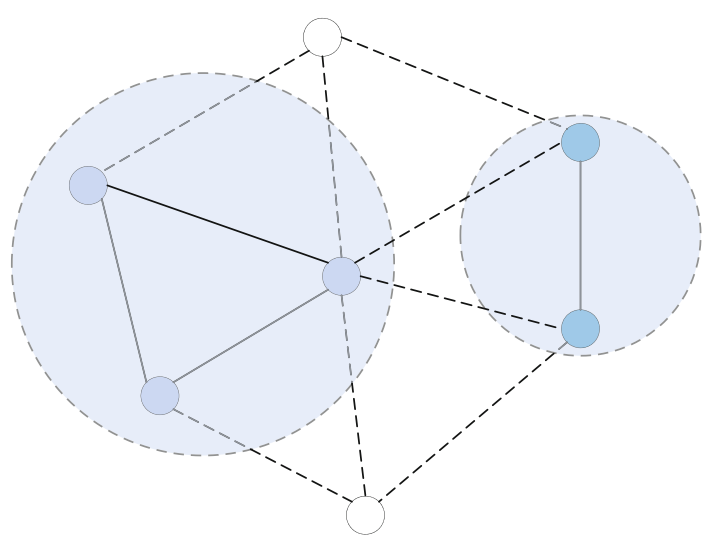

Figure 8: Connection diagram.

$H$ is called the SLFN hidden layer output matrix:

$H\left(a_{1}, \cdots, a_{\bar{N}}, b_{1}, \cdots, b_{\bar{N}}, x_{1}, \cdots, x_{N}\right)=\left[\begin{array}{ccc}g\left(a_{1} \cdot x_{1}, b_{1}\right) & \cdots & g\left(a_{\bar{N}} \cdot x_{1}, b_{\bar{N}}\right) \\ \vdots & \cdots & \vdots \\ g\left(a_{1} \cdot x_{N}, b_{1}\right) & \cdots & g\left(a_{\bar{N}} \cdot x_{N}, b_{\bar{N}}\right)\end{array}\right]_{N \times \bar{N}}$.

Among them,

$$
\begin{gathered}
\beta=\left[\begin{array}{c}
\beta_{1}^{T} \\
\vdots \\
\beta_{\bar{N}}^{T}
\end{array}\right]_{\bar{N} \times m}, \\
T=\left[\begin{array}{c}
t_{1}^{T} \\
\vdots \\
t_{N}^{T}
\end{array}\right]_{N \times m} .
\end{gathered}
$$

Formula (7) is a linear model, and the output weight can be calculated as follows:

$$
\begin{gathered}
\beta=H^{i} T, \\
H^{i}=\left(H^{T} H+\frac{1}{C}\right)^{-1} H^{T} .
\end{gathered}
$$

In the formula, $H^{i}$ is the generalized pseudoinverse of $H$. Among them,

$$
\begin{gathered}
H_{0}=\left[\begin{array}{ccc}
g\left(a_{1} \cdot x_{1}, b_{1}\right) & \cdots & g\left(a_{\bar{N}} \cdot x_{1}, b_{\bar{N}}\right) \\
\vdots & \cdots & \vdots \\
g\left(a_{1} \cdot x_{N_{0}}, b_{1}\right) & \cdots & g\left(a_{\bar{N}} \cdot x_{N_{0}}, b_{\bar{N}}\right)
\end{array}\right]_{N_{0} \bar{N}}, \\
T_{0}=\left[\begin{array}{c}
t_{1}^{T} \\
\vdots \\
t_{N_{0}}^{T}
\end{array}\right]_{N_{0} \times m},
\end{gathered}
$$

TABLE 1: Expert screening results of physical fitness test indicators $(N=6)$.

\begin{tabular}{lcccc}
\hline Body index & $\begin{array}{c}\text { Very } \\
\text { important }\end{array}$ & $\begin{array}{c}\text { Influence level } \\
\text { Generally }\end{array}$ & $\begin{array}{c}\text { Unimportant } \\
\text { Sotal } \\
\text { score }\end{array}$ \\
\hline 30 m sprint & 4 & 0 & 0 & 28 \\
12-minute run & 1 & 0 & 2 & 15 \\
Standing long & 3 & 1 & 0 & 26 \\
jump & 1 & 0 & 0 & 19 \\
Yoyo & 0 & 3 & 1 & 14 \\
1000 m & 5 & 0 & 0 & 29 \\
Back and forth & & & & \\
\hline
\end{tabular}

can be calculated:

$$
\beta^{(0)}=K_{0}^{-1} H_{0}^{T} T_{0}
$$

Among them,

$$
K_{0}=H_{0}^{T} H_{0}
$$

When a new sample enters the model, assuming there are $N_{1}$ samples, the problem becomes minimized at this time:

$$
\min _{\beta}\left\|\left[\begin{array}{l}
H_{0} \\
H_{1}
\end{array}\right] \beta-\left[\begin{array}{l}
T_{0} \\
T_{1}
\end{array}\right]\right\|
$$

According to the generalized inverse, the value of $\beta_{0}^{(1)}$ can be calculated as

$$
\begin{gathered}
\beta^{(1)}=K_{1}^{-1}\left[\begin{array}{l}
H_{0} \\
H_{1}
\end{array}\right]^{T}\left[\begin{array}{l}
T_{0} \\
T_{1}
\end{array}\right], \\
K_{1}=\left[\begin{array}{l}
H_{0} \\
H_{1}
\end{array}\right]^{T}\left[\begin{array}{l}
H_{0} \\
H_{1}
\end{array}\right] .
\end{gathered}
$$

It can be seen that the output weight becomes

$$
\begin{aligned}
& \beta^{(1)}=K_{1}^{-1}\left[\begin{array}{l}
H_{0} \\
H_{1}
\end{array}\right]^{T}\left[\begin{array}{l}
T_{0} \\
T_{1}
\end{array}\right], \\
& \beta^{(1)}=K_{1}^{-1}\left(K_{1} \beta^{(0)}-H_{1}^{T} H_{1} \beta^{(0)}+H_{1}^{T} H_{1}\right), \\
& \beta^{(1)}=\beta^{(0)}+K_{1}^{-1} H_{1}^{T}\left(T_{1}-H_{1} \beta^{(0)}\right),
\end{aligned}
$$

to make

$$
P_{k+1}=K_{k+1}^{-1} \text {. }
$$




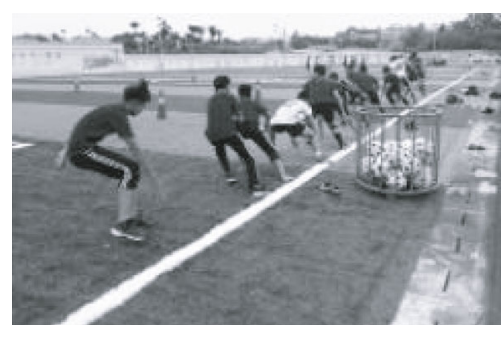

(a) Turn-back running test site

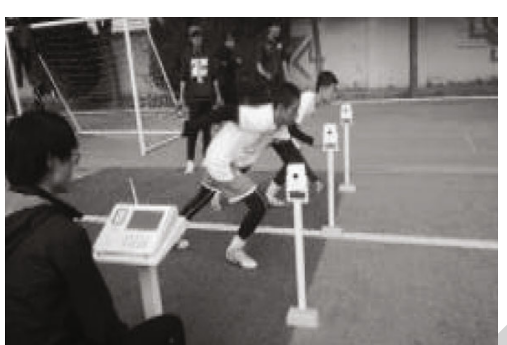

(b) Physical fitness test site

FIgURE 9: Turn-back run and physical fitness assessment site.

TABLE 2: Football technical teaching ability score sheet.

\begin{tabular}{|c|c|c|c|c|c|}
\hline Content & Full marks & Excellent & Good & Medium & Difference \\
\hline Skill and tactic teaching & 5 & 5 & 4 & 3 & $2-1$ \\
\hline Teaching attitude & 5 & 5 & 4 & 3 & $2-1$ \\
\hline Technical action explanation & 8 & 8 & $7-6$ & $5-4$ & $3-1$ \\
\hline Teaching plan & 5 & 5 & 4 & 3 & $2-1$ \\
\hline Formation transfer & 5 & 5 & 4 & 3 & $2-1$ \\
\hline Teaching effect & 5 & 5 & 4 & 3 & $2-1$ \\
\hline Total & 33 & - & - & - & - \\
\hline
\end{tabular}

From this, we can get

$$
\beta^{(k+1)}=\beta^{(k)}+P_{k+1} H_{k+1}^{T}\left(T_{k+1}-H_{k+1} \beta^{(k)}\right) .
$$

OR-ELM can improve prediction performance by updating input weights and output weights. The algorithm is divided into an initialization phase and an online cycle learning phase. In the initialization stage, the output weight $\beta_{0}$ of the initial input data with $N_{0}$ training samples is calculated as follows:

$$
\beta_{0}=P_{0} H_{0} T_{0}, P_{0}=\left(H_{0}^{T} H_{0}+\frac{I \mid}{C}\right)^{-1}
$$

In the online cyclic learning stage, whenever $N_{K+1}$ new sample input data arrives, the output weight is updated:

$$
\begin{gathered}
\beta_{k+1}=\beta_{k}+P_{k+1} H_{k+1}^{T}\left(T_{k+1}-H_{k+1} \beta_{k}\right), \\
P_{k+1}=P_{k}-P_{k} H_{k+1}^{T}\left(I+H_{k+1} P_{k} H_{k+1}^{T}\right)^{-1} H_{k+1} P_{k} .
\end{gathered}
$$

Among them, $K+1$ represents the $K+1$ input data block increased from $k$, and $H_{K+1}$ represents the hidden layer output of the $K+1$ input data block. The forgetting factor $\lambda$ can further improve the short-term prediction accuracy:

$$
P_{k+1}=\frac{1}{\lambda} P_{k}-P_{k} H_{k+1}^{T}\left(I+H_{k+1} P_{k} H_{k+1}^{T}\right)^{-1} H_{k+1} P_{k}
$$

Among them, $\lambda \in(0,1]$; then, use the activation function to update the input weights and input samples:

$$
x(k+1) \in R^{n \times 1} .
$$

TABLE 3: Pretest results of physical fitness of the test group and the control group.

\begin{tabular}{lcccc}
\hline Test index & Test group & $\begin{array}{c}\text { Control } \\
\text { group }\end{array}$ & $\begin{array}{c}T \\
\text { value }\end{array}$ & $\begin{array}{c}P \\
\text { value }\end{array}$ \\
\hline $30 \mathrm{~m} \mathrm{(s)}$ & $4.41 \pm 0.07$ & $4.35 \pm 0.08$ & 1.764 & 0.087 \\
Back and forth & $33.68 \pm 1.14$ & $33.07 \pm 1.31$ & 1.846 & 0.071 \\
$\begin{array}{l}\text { Standing long } \\
\text { jump }\end{array}$ & $2.64 \pm 0.07$ & $2.69 \pm 0.76$ & -1.561 & 0.127 \\
\hline
\end{tabular}

TABle 4: Pretest results of football learning interest in the test group and the control group.

\begin{tabular}{lcccc}
\hline Dimension & Test group & Control group & $T$ value & $P$ value \\
\hline Active interest & $18.67 \pm 2.38$ & $19.87 \pm 1.87$ & -0.234 & 0.816 \\
Negative interest & $20.08 \pm 1.84$ & $20.11 \pm 1.34$ & -0.278 & 0.749 \\
Participation & $18.42 \pm 1.07$ & $18.24 \pm 1.62$ & 0.429 & 0.647 \\
Autonomy & $18.33 \pm 2.39$ & $18.07 \pm 1.58$ & 0.428 & 0.624 \\
Attention & $16.29 \pm 1.59$ & $17.34 \pm 1.74$ & -0.846 & 0.431 \\
\hline
\end{tabular}

TABLE 5: Comparison results of the physical fitness of the test group before and after the experiment.

\begin{tabular}{lcccc}
\hline Test index & Test group & $\begin{array}{c}\text { Control } \\
\text { group }\end{array}$ & $\begin{array}{c}T \\
\text { value }\end{array}$ & $\begin{array}{c}P \\
\text { value }\end{array}$ \\
\hline $30 \mathrm{~m}$ & $4.38 \pm 0.06$ & $4.36 \pm 0.06$ & 1.842 & 0.084 \\
Back and forth & $33.69 \pm 1.11$ & $34.11 \pm 0.92$ & -0.719 & 0.469 \\
$\begin{array}{l}\text { Standing long } \\
\text { jump }\end{array}$ & $2.66 \pm 0.07$ & $2.71 \pm 0.08$ & -1.812 & 0.085 \\
\hline
\end{tabular}


TABLE 6: Comparison results of football skills before and after the experiment in the test group.

\begin{tabular}{lllll}
\hline Content & Test group & Control group & $T$ value & $P$ value \\
\hline $\begin{array}{l}\text { Test group } \\
\text { Bump the ball }\end{array}$ & $2.37 \pm 0.97$ & $6.79 \pm 1.71$ & -13.717 & $P<0.01$ \\
$\quad$ Shot & $4.07 \pm 0.94$ & $7.49 \pm 1.28$ & -11.707 & $P<0.01$ \\
Control group & & & & \\
$\quad$ Bump the ball & $2.74 \pm 1.16$ & $5.24 \pm 1.07$ & -11.168 & $P<0.01$ \\
$\quad$ Shot & $4.27 \pm 1.31$ & $6.27 \pm 1.12$ & -6.319 & $P<0.01$ \\
\hline
\end{tabular}

When it is propagated to the hidden layer, the hidden layer output matrix $H_{k+1}$ is calculated as follows:

$$
H_{k+1}^{i}=g\left(\operatorname{norm}\left(W_{k+1}^{i} x(k+1)\right)\right) \text {. }
$$

Among them,

$$
\begin{gathered}
\operatorname{norm}(x)=\frac{x-u^{i}}{\sqrt{\left(\sigma^{i}\right)^{2}+\varepsilon}}, \\
u^{i}=\frac{1}{L} \sum_{j=1}^{L} x_{j}, \\
\sigma^{i}=\frac{1}{L} \sum_{j=1}^{L}\left(x_{j}-u_{i}\right)^{2} .
\end{gathered}
$$

This algorithm is a data fusion algorithm based on multimedia network and only needs a small amount of historical data to train the model. In real-time data fusion, the model can update the parameters online and adjust the weight coefficients adaptively to obtain higher accuracy. The fusion mechanism runs on the sensor node and the terminal at the same time.

\section{Flipped Classroom Controlled Experiment}

3.1. Selection and Assessment of Experimental Indicators. For this reason, this article would like to further study it and explore whether this model can have a positive impact in sports professional football teaching. Based on the concept of flipped classroom teaching and combined with previous research results, this article selects the following indicators for experimental research:

\section{(1) Physical fitness}

Through the selection of the expert questionnaire, the first three items with the highest weight coefficients are selected as the test indicators of physical fitness in this experiment. After screening, the $5 * 25$-meter reentry run, the 30 meter sprint, and the standing long jump are used as physical fitness test indicators. The specific conditions are shown in Table 1.
The test content included three indicators: 30-meter sprint, standing long jump, and $5 * 25$-meter return run. The 30-meter sprint can reflect the quality of speed; the 5 $* 25$-meter retracement can reflect the physical quality of speed, endurance, and agility. Figure 9 shows the $5 * 25 \mathrm{~m}$ turn-back run test and physical fitness assessment site of the test group.

(2) Football teaching ability

In the last week of the experiment, the students' football technique teaching ability was evaluated. According to the assessment requirements, each student's teaching time is 15 minutes, and the teaching format is simulated class. The teaching content is the basic part of the class, including the explanation and demonstration of football skills, the teaching process, and the organization and mobilization of the team. There are 10 students in each class, and the assessment is divided into two classes. The technical teaching assessments of the two classes are performed by two football teachers from the Football Teaching and Research Section of the Shanghai Institute of Physical Education. According to the teaching design of the whole class, the use of field equipment, teaching postures, and teaching attitudes, the teacher comprehensively evaluates the students' basic football skill teaching situation. The football skill teaching ability scores are shown in Table 2:

3.2. Pretest Results and Analysis. The pretest results and analysis of physical fitness show that good physical fitness is the foundation and guarantee of football, and it is also an important part of football teaching. The students in the two classes were tested for 30 -meter run, $5 * 25$ reentry running, and standing long jump before the experiment. The results are shown in Table 3.

The 30-meter score of the control group students was $4.37 \pm 0.06 \mathrm{~s}$, the $5 * 25$ foldback running score was 33.09 $\pm 1.27 \mathrm{~s}$, and the standing long jump was $2.70 \pm 0.07 \mathrm{~m}$. The results showed that $P 30 \mathrm{~m}=0.083>0.05, P$ reentry running $=0.072>0.05, P$ standing long jump $=0.13>0.05$.

\subsubsection{Pretest Results and Analysis of Football Learning} Interest. The results are shown in Table 4.

The main reason for the analysis is that the traditional teaching mode of physical education is deeply ingrained in students' minds. Students think that football lessons are as boring as other physical education lessons, which affects students' cognition of football. In addition, the students in the two classes seldom touched the football project before this, so it will cause the students to be less interested in football learning.

\subsection{Posttest Results and Analysis}

3.3.1. Posttest Results and Analysis of Physical Fitness. In the last week of the experiment, the same as the previous test, the two classes of students' 30 -meter run, $5 * 25$ reentry run, and standing long jump were tested to test whether the football flipped classroom teaching model has an impact on the students' physical fitness. The comparative data of 

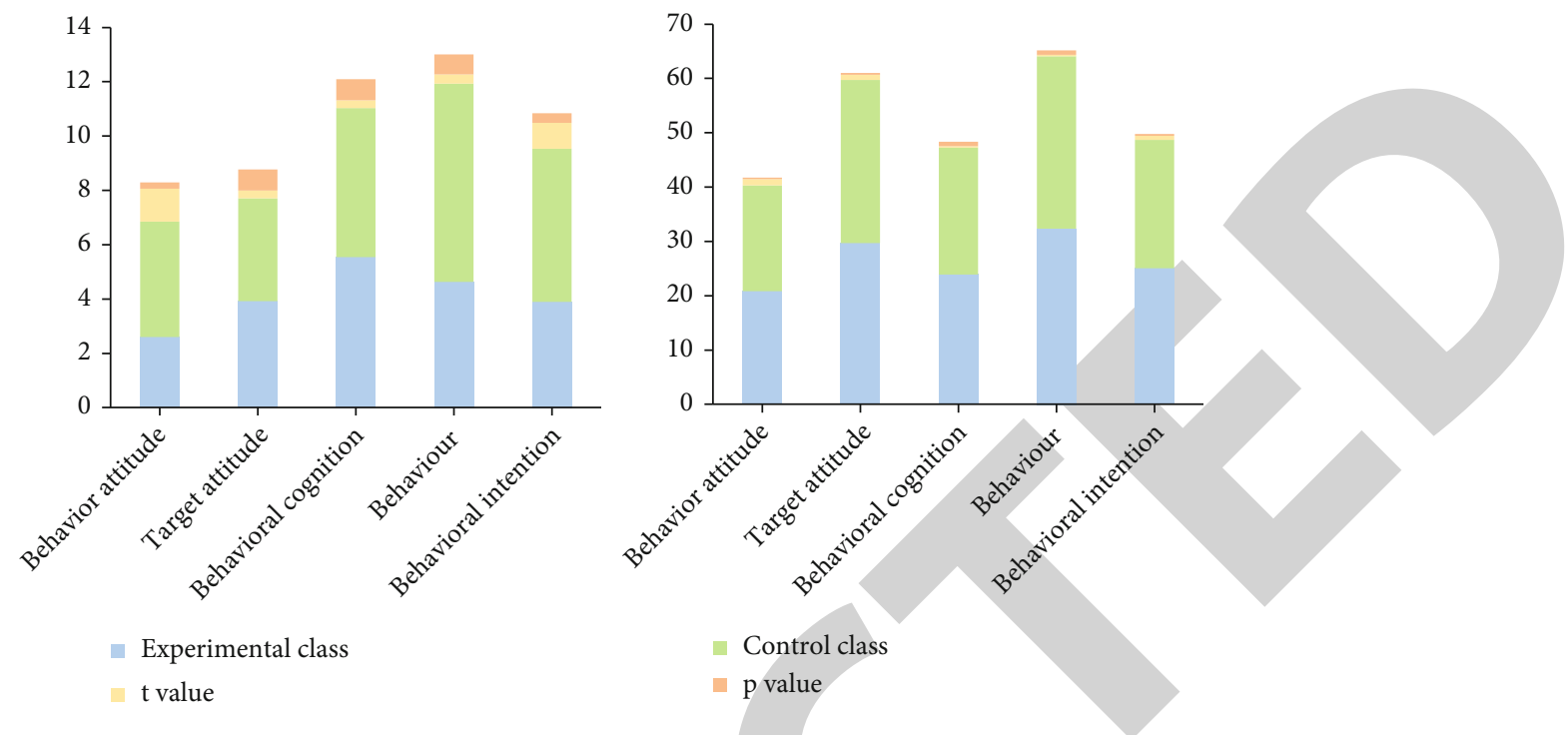

Figure 10: Table of comparative analysis of the test results of the physical exercise attitude of the test group and the control group before the experiment.

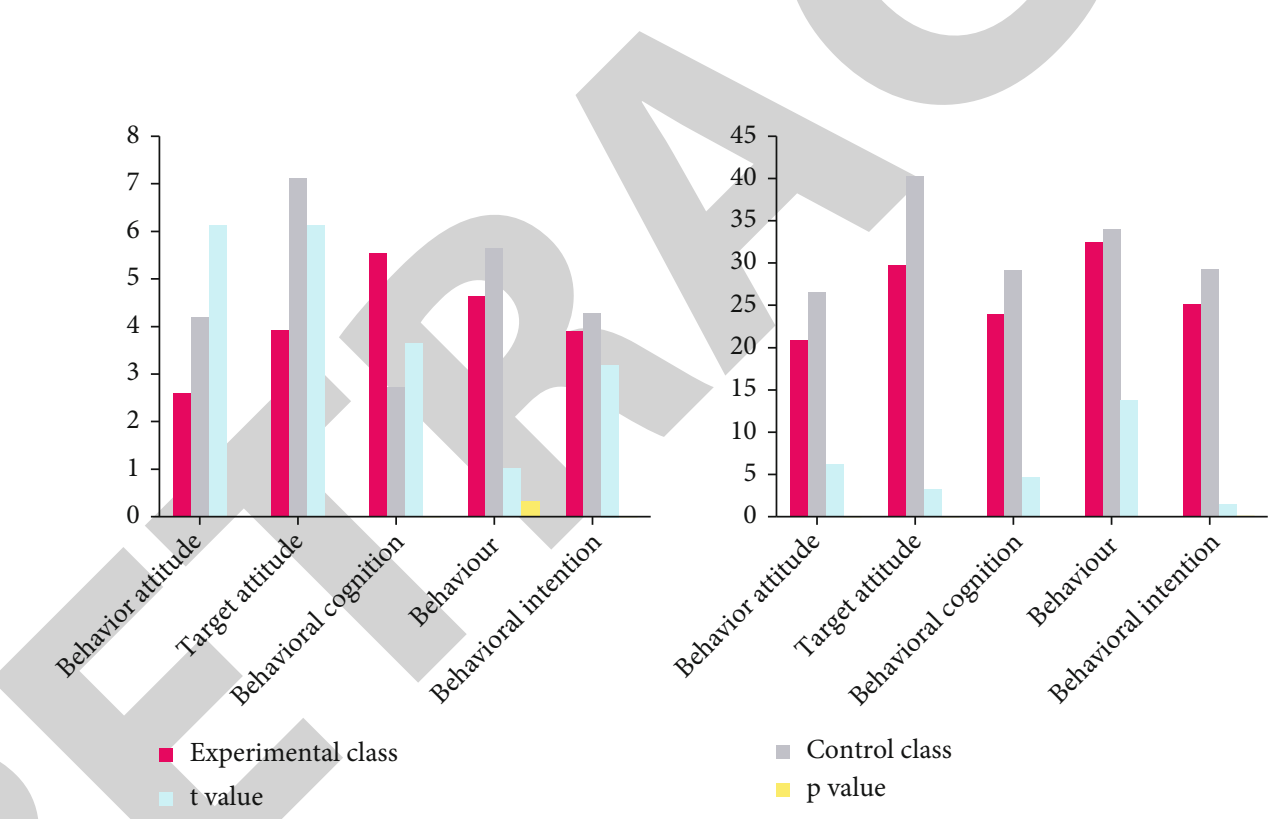

FIGURE 11: Table of comparative analysis of the test results of physical exercise attitude test before and after the experiment in the test group.

physical fitness before and after the experiment is shown in Table 5.

Table 5 shows that after the experiment, the three indicators of physical fitness before and after the experiment of the test group were, respectively, subjected to a paired sample $T$ test, and the results were $P 30 \mathrm{~m}=0.083$ $>0.05, P$ reentry running $=0.475>0.05$, and $P$ standing long jump $=0.087>0.05$.

3.3.2. Posttest Results and Analysis of Football Skills. Football skill level is an important indicator. The comparison results are shown in Table 6.

\section{Comparative Analysis of the Test Results of the Physical Exercise Attitude of the Test Group and the Control Group}

4.1. Comparative Analysis of the Physical Exercise Attitude of the Two Classes of Students

4.1.1. Comparative Analysis of the Physical Exercise Attitudes of the Two Classes of Students before the Experiment. The physical exercise attitude scale is mainly borrowed from "Manual for the Evaluation of Sports Science Commonly Used Mental Scales." Adding the scores of all the questions 

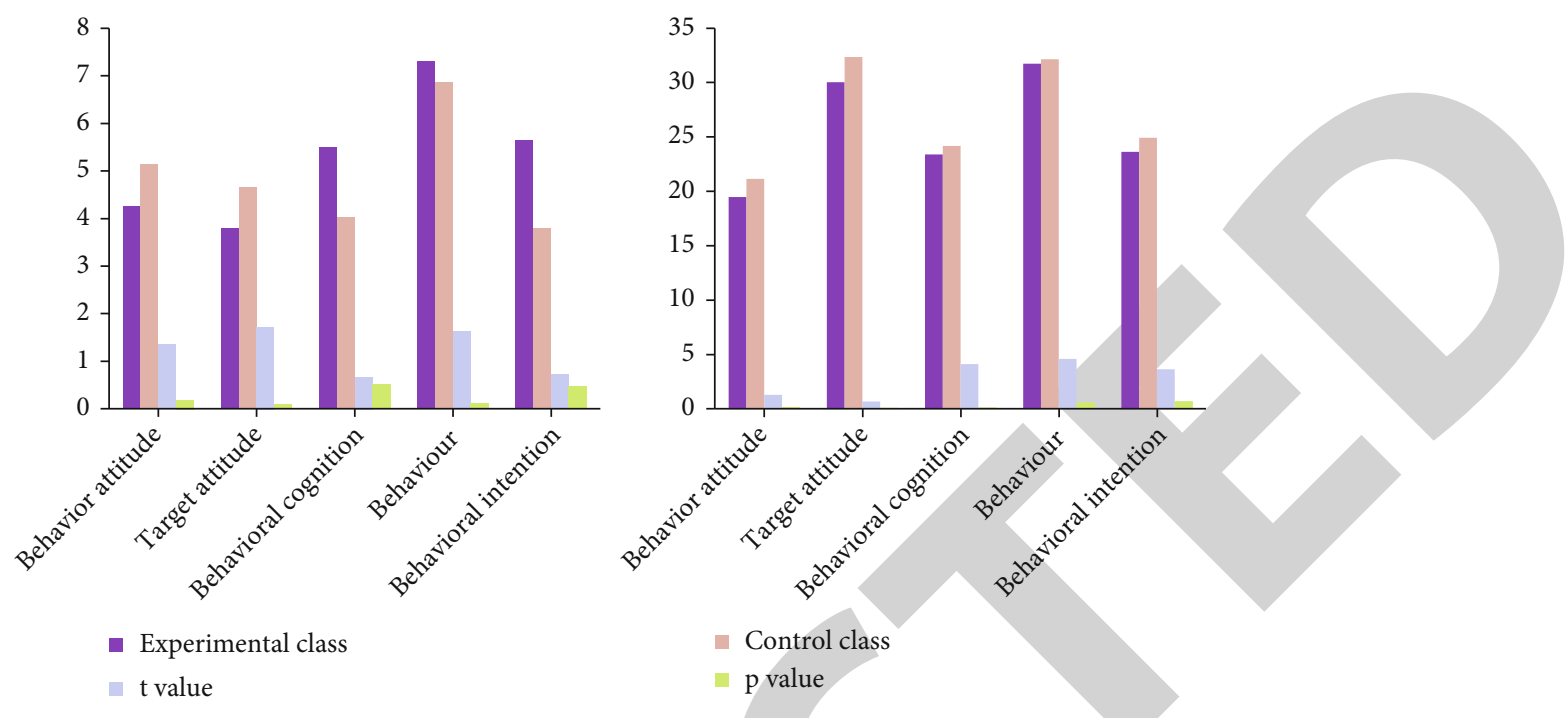

Figure 12: Comparative analysis of the results of the physical exercise attitude test results of the control group before and after the experiment.

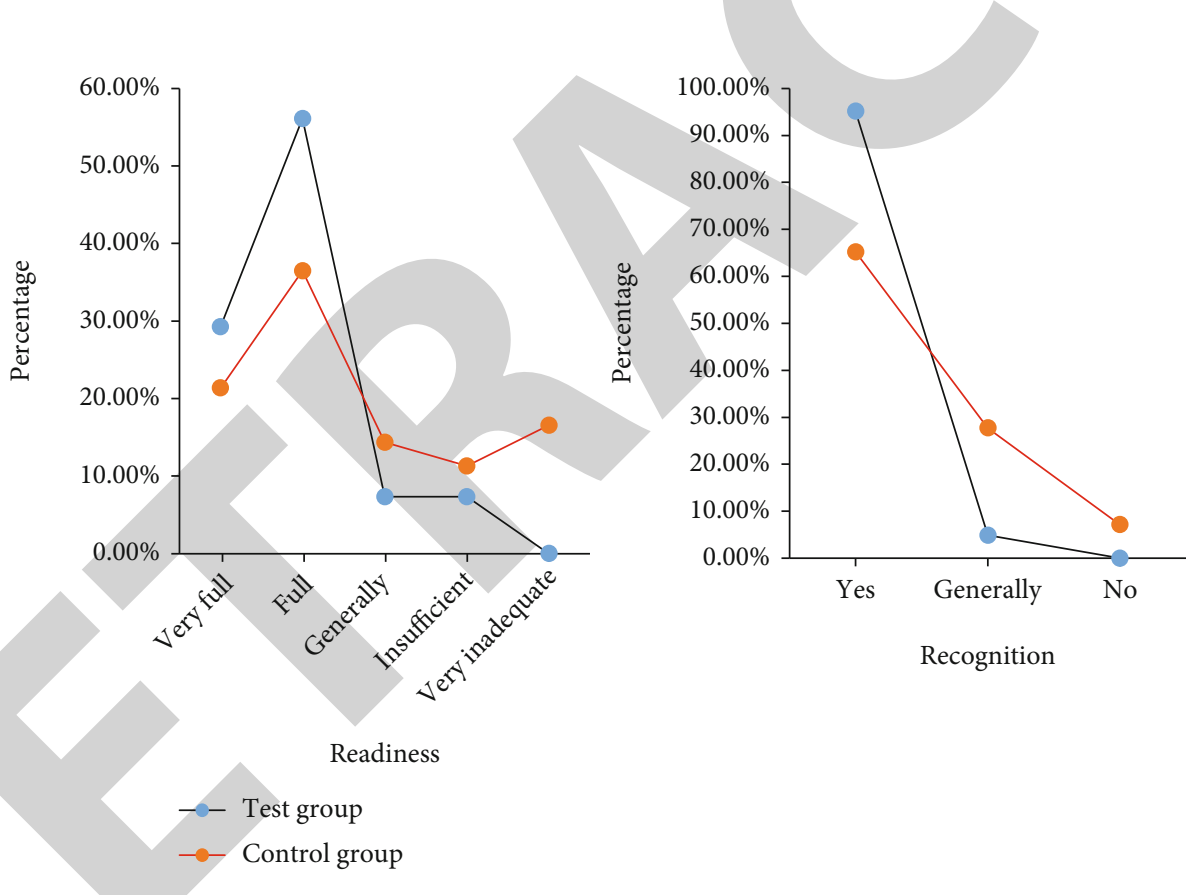

FIGURE 13: The experimental group and the control group's exercise status and recognition of the flipped classroom.

is the total score of the subjects, and the level of the scores represents the level of the subjects' autonomous learning ability. The attitude test results of the test group and the control group before the experiment are shown in Figure 10.

After comparative analysis, the mean $(M)$, standard deviation (SD), and corresponding $t$ value and $P$ value of the 8 latitude test results of physical exercise attitude of the test group and the control group are obtained. Independent samplet-test was used to test the results of students' attitude towards physical exercise learning in experimental class and control class before the experiment.

4.2. A Comparative Analysis of the Physical Exercise Attitudes of the Two Classes of Students after the Experiment. In order to study the physical exercise situation of the students in the test group and the control group after the experiment, at the end of the experiment, the attitude of the students in the test group and the control group was retested.

Compare the two sets of data, and compare the changes before and after their attitudes which are shown in Figure 11.

At the same time, the test group and the control group are compared with the test data before and after the experiment, and the paired sample $T$ test is used to reflect the change of the physical exercise attitude of the control group before and after the experiment, as shown in Figure 12.

After analysis and comparison, it is found that the control group has no obvious change in the attitude of physical exercise, and the test group has adopted the "flipped 
classroom" teaching method, so the change is more obvious. The "flipped classroom" teaching method has advanced teaching methods and unique methods, which fully tap students' autonomous learning ability, can use a relatively short time, enable students to efficiently complete the teaching content requirements, and promote the development of students' self-discipline.

In summary, the "flipped classroom" teaching method used in the test group can improve students' attitudes to physical exercise more than traditional teaching methods, and the effect is more significant, and it also promotes the development of student behavior.

4.3. Value Analysis of the Introduction of Flipped Classrooms into College Public Physical Education. "Health first" is the guiding ideology of China's physical education, which is beyond doubt. However, under the traditional physical education teaching model, explanations and demonstrations occupy most of the classroom time, and there is a serious shortage of time for students to explore and practice. Under this circumstance, it is difficult to achieve the purpose of improving students' physical health, mental health, and social adaptability, so the guiding ideology of "health first" has been questioned by many quarters. Figure 13 shows the movement status and cognition of the experimental group and control group students in the flipped classroom.

From the above analysis and combined with the results of physical education teaching experiments, it can be seen that under the flipped classroom mode, students are significantly better than the traditional physical education mode in terms of skill mastery and improvement of attitudes towards exercise. This fully proves that the implementation of flipped classrooms in public physical education in Chinese universities can help improve classroom effectiveness.

After the above experimental analysis, we can see that the college sports flipped classroom after the fusion of multimedia network data improves the students' autonomous learning ability by $31 \%$, learning interest has increased by $28 \%$, physical fitness after applying this model has increased by $14 \%$, and related sports skills have also been improved to varying degrees. It can be seen that under this mode, students' interest in learning has been fully mobilized, which has greatly subverted the past phenomenon of college physical education classrooms.

\section{Conclusions}

This article is mainly to study the flipped teaching of college physical education, through the use of multimedia network data fusion, to monitor college students' physical classroom activities to a certain extent. The related data was measured, and experiments and analysis were designed to perform fusion analysis on the obtained data. The analysis results showed that the enthusiasm of the students in the improved education model was greatly mobilized. College students' physical education classes are no longer the way they were doing nothing in the past, but they started to learn and exercise with interest. This phenomenon can be said to be rare in college physical education classes, and the physical fitness of students can be improved to varying degrees after exercise.

\section{Data Availability}

No data were used to support this study.

\section{Conflicts of Interest}

The authors declare that there is no conflict of interest with any financial organizations regarding the material reported in this manuscript.

\section{References}

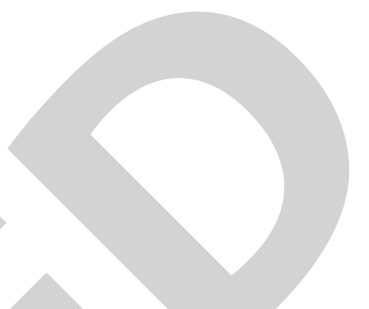

[1] M. Z. Hasan, H. Al-Rizzo, and F. Al-Turjman, "A survey on multipath routing protocols for QoS assurances in real-time wireless multimedia sensor networks," Communications Surveys \& Tutorials IEEE, vol. 19, no. 3, pp. 1424-1456, 2017.

[2] S. W. Park, C. H. Cho, and S. B. Choi, "Social multimedia network service quality, user satisfaction, and prosumer activity," Multimedia Tools \& Applications, vol. 76, no. 16, pp. 1721317229, 2017.

[3] F. Al-Turjman and A. Radwan, "Data delivery in wireless multimedia sensor networks: challenging and defying in the IoT era," IEEE Wireless Communications, vol. 24, no. 5, pp. 126$131,2017$.

[4] C. F. Lai, Y. X. Lai, M. S. Wang, and J. W. Niu, "An adaptive energy-efficient stream decoding system for cloud multimedia network on multicore architectures," IEEE Systems Journal, vol. 8, no. 1, pp. 194-201, 2014.

[5] J. Jovanović, D. Gašević, S. Dawson, A. Pardo, and N. Mirriahi, "Learning analytics to unveil learning strategies in a flipped classroom," The Internet and Higher Education, vol. 33, no. 4, pp. 74-85, 2017.

[6] H. Baytiyeh, "The flipped classroom model: when technology enhances professional skills," International Journal of Information \& Learning Technology, vol. 34, no. 1, pp. 5162, 2017.

[7] B. McNally, J. Chipperfield, P. Dorsett et al., "Flipped classroom experiences: student preferences and flip strategy in a higher education context," Higher Education, vol. 73, no. 2, pp. 281-298, 2017.

[8] C. Kostaris, S. Stylianos, D. G. Sampson, M. Giannakos, and L. Pelliccione, "Investigating the potential of the flipped classroom model in K-12 ICT teaching and learning: an action research study," Educational Technology \& Society, vol. 20, no. 1, pp. 261-273, 2017.

[9] J. H. Park, "Advances in algorithm, multimedia, and network for future IT," Journal of Information Processing Systems, vol. 13, no. 2, pp. 197-203, 2017.

[10] Y. S. Kavian, H. Sharif, and D. Peng, "Editorial note: Wireless multimedia sensor networks," Multimedia Tools \& Applications, vol. 77, no. 4, article 4431, 2018.

[11] Z. Lv and H. Song, "Trust mechanism of feedback trust weight in multimedia network," ACM Transactions on Multimedia Computing, Communications, and Applications, vol. 17, no. 4, pp. 1-26, 2021.

[12] J. Huang, H. Wang, and Q. Yi, "Game user-oriented multimedia transmission over cognitive radio networks," IEEE 
Transactions on Circuits \& Systems for Video Technology, vol. 27, no. 1, pp. 198-208, 2017.

[13] C. Lo and K. Hew, "Using "First Principles of Instruction" to design secondary school mathematics flipped classroom: the findings of two exploratory studies," Journal of Educational Technology \& Society, vol. 20, no. 1, pp. 222-236, 2017.

[14] C. Chi, "An investigation of the use of the'flipped classroom'pedagogy in secondary English language classrooms," Journal of Information Technology Education Innovations in Practice, vol. 16, no. 1, pp. 1-20, 2017.

[15] F. S. Sadia, A. F. Manzoor, and E. S. Ather, "Flipped classroom instructional approach in undergraduate medical education," Pakistan Journal of Medical Sciences, vol. 33, no. 6, pp. 14241428, 2017.

[16] S. Sergis, D. G. Sampson, and L. Pelliccione, "Investigating the impact of flipped classroom on students' learning experiences: a self-determination theory approach," Computers in Human Behavior, vol. 78, pp. 368-378, 2018.

[17] F. H. Wang, "An exploration of online behaviour engagement and achievement in flipped classroom supported by learning management system," Computers \& Education, vol. 114, pp. 79-91, 2017.

[18] H. Mohamed and M. Lamia, "Implementing flipped classroom that used an intelligent tutoring system into learning process," Computers \& Education, vol. 124, pp. 62-76, 2018.

[19] N. O. García, M. F. Velásquez, C. A. Romero, J. H. Ortiz, and O. I. Khalaf, "Remote academic platforms in times of a pandemic," International Journal of Emerging Technologies in Learning, vol. 16, no. 21, pp. 121-131, 2021.

[20] J. Y. Hong, H. Ko, L. Mesicek, and M. B. Song, "Cultural intelligence as education contents: exploring the pedagogical aspects of effective functioning in higher education," Concurrency and Computation Practice and Experience, vol. 33, article e5489, 2021.

[21] Z. Zainuddin, "First-year college Students' experiences in the EFL flipped classroom: a case study in Indonesia," International Journal of Instruction, vol. 10, no. 1, pp. 133-150, 2017.

[22] A. K. Singh, X. Liu, and H. Wang, "Recent advances in multimedia security and information hiding," Transactions On Emerging Telecommunications Technologies, vol. 32, no. 2, article e4193, 2021.

[23] S. N. Alsubari, S. N. Deshmukh, A. A. Alqarni et al., "Data analytics for the identification of fake reviews using supervised learning," CMC-Computers, Materials \& Continua, vol. 70, no. 2, pp. 3189-3204, 2022.

[24] S. Palanisamy, B. Thangaraju, O. I. Khalaf, Y. Alotaibi, and S. Alghamdi, "Design and synthesis of multi-mode bandpass filter for wireless applications," Electronics, vol. 10, p. 2853, 2021. 